\title{
Increased brood size leads to persistent eroded telomeres
}

\section{Sophie Reichert ${ }^{1,2 *}$, Antoine Stier ${ }^{1,2}$, Sandrine Zahn ${ }^{1,2}$, Mathilde Arrivé ${ }^{1,2}$, Pierre Bize $^{3}$, Sylvie Massemin ${ }^{1,2 t}$ and François Criscuolo ${ }^{1,2 t}$}

\author{
1 Université de Strasbourg, Institut Pluridisciplinaire Hubert Curien, Département Ecologie, Physiologie et Ethologie, Strasbourg, France \\ ${ }^{2}$ CNRS, UMR 7178, Strasbourg, France \\ ${ }^{3}$ School of Biological Sciences, University of Aberdeen, Aberdeen, Scotland, UK
}

\section{Edited by:}

Patrick S. Fitze, University of

Lausanne, Switzerland

Reviewed by:

Morgan David, University of

Antwerp, Belgium

Carlos Alonso Alvarez, Consejo

Superior de Investigaciones

Cientificas, Spain

${ }^{*}$ Correspondence:

Sophie Reichert, Institut

Pluridisciplinaire Hubert Curien, Département Ecologie, Physiologie et Ethologie, 23 rue Becquerel, 67087 Strasbourg Cedex 2, France e-mail: reichert.sophie@gmail.com

these authors have contributed equally to this work.
Costs of reproduction can be divided in mandatory costs coming from physiological, metabolic, and anatomical changes required to sustain reproduction itself, and in investment-dependent costs that are likely to become apparent when reproductive efforts are exceeding what organisms were prepared to sustain. Interestingly, recent data showed that entering reproduction enhanced breeders' telomere loss, but no data explored so far the impact of reproductive investment. Telomeres protect the ends of eukaryote chromosomes. Shortened telomeres were associated with shorter lifespan, telomere erosion being then proposed to powerfully quantify life's insults. Here, we experimentally manipulated brood size in order to modify reproductive investment of adult zebra finches citation(Taeniopygia guttata) below or beyond their citation(optimal) starting investment and tested the consequences of our treatment on parents' telomere dynamics. We show that an increased brood size led to a reduction in telomere lengths in both parents compared to control and to parents raising a reduced brood. This greater telomere erosion was detected in parents immediately after the reproductive event and the telomere length difference persisted up to 1 year later. However, we did not detect any effects of brood size manipulation on annual survival of parents kept under laboratory conditions. In addition, telomere lengths at the end of reproduction were not associated with annual survival. Altogether, although our findings highlight that fast telomere erosion can come as a cost of brood size manipulation, they provide mixed correlative support to the emerging hypothesis that telomere erosion could account for the links between high reproductive investment and longevity.

\section{Keywords: aging, cost of reproduction, clutch size manipulation, telomere, oxidative stress, bird, zebra finch}

\section{INTRODUCTION}

A central tenet of life history theory is that reproduction can come as a cost of longevity. Accordingly, numerous empirical studies demonstrated that individuals prevented from reproduction live longer than those allowed to reproduce (Smith, 1958; Partridge and Farquhar, 1981) and that an increase in parental reproductive effort can "precipitate death" (Daan et al., 1996; Santos and Nakagawa, 2012). The underlying mechanisms accounting for costs of reproduction on longevity remain however little understood and are the current topic of intense investigations (Harshman and Zera, 2007; Speakman, 2008; Monaghan et al., 2009).

Costs of reproduction can be divided in two complementary components. Firstly, reproduction itself is associated with numerous, mandatory, changes that stem chiefly from changes in sex hormone levels and of their pleiotropic effects on traits as diverse as immunity, metabolism, and behaviors (Brown-Borg, 2007). Experimental manipulation of sex hormone profiles, for instance using castration in males and progesterone implants in females, are supporting the existence of mandatory costs of engagement in reproduction on longevity (Tavecchia et al., 2005; Brown-Borg, 2007; Min et al., 2012). Secondly, once reproduction has started, parents of species that display parental care to their offspring are expected to optimize their reproductive investment according to their own condition citation(or quality) and to environmental conditions encountered during reproduction (Stearns, 1992; Santos and Nakagawa, 2012). This optimization problem is commonly studied in birds by manipulating clutch size or brood size, and in so doing parental effort (Santos and Nakagawa, 2012). Accumulating evidence demonstrates that parental costs of reproduction on longevity are "investment-dependent," becoming apparent only when reproductive efforts are exceeding what parents were prepared to sustain (Santos and Nakagawa, 2012).

Interestingly, although there is little doubt nowadays that reproduction can shorten lifespan through combinations of mandatory and investment-dependent costs, one emerging hypothesis is that reproduction impacts adult survival and/or longevity through a shared, endpoint, aging mechanism: telomere erosion (Allsopp et al., 1992; Cawthon et al., 2003; Bize et al., 2009).

Telomeres are highly conserved non-coding repetitive DNA sequences located at the end of linear chromosomes which hide the chromosome ends as being recognized as double stranded breaks (Blackburn, 1991). Because of the inability of telomeres to be completely replicated by DNA polymerase, progressive telomere shortening occurs over cell divisions. Once reaching a lower 
critical size, telomere signalization pathway induces cell division arrest, and/or cell senescence (Harley et al., 1990; Blackburn, 1991). Furthermore, the rate at which telomeres are lost is dependent on the balancing of pro- and anti-erosion factors. For instance, oxidative stress comes from the imbalance between the production of reactive oxygen species and the antioxidant capacity (Finkel and Holbrook, 2000). Actually, increased reproductive effort has been previously associated with decreased protection against oxidative stress (Wiersma et al., 2004) or with altered cell resistance to an oxidative burst (Alonso-Alvarez et al., 2004). However, whether reproduction causes oxidative stress remains an open question and can only be answered using experimental approaches (i.e., brood size manipulation) (Stier et al., 2012; Metcalfe and Monaghan, 2013).

Oxidative stress accelerates telomere loss (Von Zglinicki, 2002; Haussmann et al., 2011; Blackburn and Epel, 2012) while telomerase activity (mainly active in germinal and stem cells) and shelterin protein complex stability favor telomere maintenance (Blackburn, 2000; De Lange, 2009). Therefore, telomeres are not just cell division counters but their rate of erosion is actually largely modulated by environmental stressors thereby explaining the age-matched individual variability observed in telomere length (Haussmann et al., 2003; Hall et al., 2004; Monaghan and Haussmann, 2006). This telomere ability to "powerfully quantify life's insults" (Blackburn and Epel, 2012) makes them a potential proxy of future individual fitness, which starts now to be confirmed by the accumulation of an increasing number of studies, from humans to birds (Cawthon et al., 2003; Haussmann et al., 2005; Pauliny et al., 2006; Bize et al., 2009; Salomons et al., 2009; Heidinger et al., 2012; Bauch et al., 2013).

One of the main results of (Heidinger et al., 2012) study was that "engaging in reproduction accelerated telomere shortening." However, this effect was not related to the number of reproductive events undertaken during this time period, and reproductive birds did not exhibit shorter telomeres than non-reproducters after 2 years. This is a rather surprising result in the light of the expected trade-off between reproduction and adult lifespan [see (Kotrschal et al., 2007) for a suggested link between reproduction and telomere loss]. One important point was that birds were only allowed to lay eggs and not to raise chicks during each reproductive attempt (Heidinger et al., 2012), therefore largely reducing reproductive costs due to brooding and chick feeding. Hence, one explanation may be that entering in the reproductive life induces sexual maturation costs, but that subsequent reproductive efforts are optimized in relation to telomere maintenance ability. If this hypothesis is right, finding a correlation between reproduction investment and telomere erosion in non-manipulated breeders, moreover under ad libitum feeding conditions will prove to be difficult. Adult reproductive investment and its impact on telomeres has been previously tested in two wild bird species. In the Adélie penguin (Pygoscelis adeliae), experimental increased reproductive effort (breeding workload being modulated through an increase of the adult foraging costs) was found to be associated with enhanced antioxidant defenses but with no changes in telomere length or survival (Beaulieu et al., 2011). Alternatively, observations in the common tern (Sterna hirundo) showed that parents providing care to their brood beyond the 10th nestling day experienced greater telomere loss compared to parents that failed in their reproduction (Bauch et al., 2013). Thus, evidence of costs of reproduction on telomere dynamics remains scarce and controversial.

To fully understand how telomere erosion is implicated in the trade-off between reproduction and longevity, we need to investigate how the level of reproductive effort during a single breeding event is affecting adult telomere loss and over which time window. To address this question, we experimentally manipulate zebra finches (Taeniopygia guttata) reproductive effort by increasing brood size and by determining the short- (end of reproduction) and mid-terms (1 year) impact on adult telomere loss. If investment in reproduction is done at the expense of longevity, we expect that pro-ageing markers (oxidative damage and telomere loss) to be increased in adult zebra finches that raised enlarged broods. To assess the effects of reproductive effort on ageing markers, we measured telomere length by relative $\mathrm{qPCR}$, as well as plasmatic oxidative stress and DNA damage. Since telomere loss is further exacerbated by exposure to oxidative stress, at least in vitro (Von Zglinicki, 2002), the oxidative balance is a good candidate to mediate the relationship between telomere dynamics and the reproduction-ageing investment trade-offs. Therefore, we also tested whether oxidative stress was involved in the potential reproduction telomere loss relationship. If increased reproductive investment has persistent effects on telomere length (even more under ad libitum feeding conditions), this may suggest that telomere loss during an unexpectedly costly breeding attempt would potentially have fitness consequences. Thus, we also checked brood size manipulation, oxidative stress, and telomere length relationships with adult survival 1 year after the reproductive event.

\section{EXPERIMENTAL PROCEDURES GENERAL PROCEDURES: BROOD SIZE MANIPULATION}

The study was conducted on captive zebra finches. We experimentally modified reproductive effort using a combined partial cross-fostering and brood size manipulation protocol. Twentyfive randomly formed pairs were placed in breeding cages $(0.57 \times$ $0.31 \times 0.39 \mathrm{~m}$ ) with food (a commercial mix of seeds for exotic birds enriched with vitamins and eggs) and water ad libitum. The cage placement within the room was random with regard to the treatment. Nest boxes and straw were provided for breeding. The cages were put in a room with a constant temperature of $24^{\circ} \mathrm{C}$ $\left( \pm 1^{\circ} \mathrm{C}\right)$ and light conditions were 13L: $11 \mathrm{D}$. Chicks from 1 to 3 days old were randomly cross-fostered (half of the chicks in each brood came from another brood) to form 9 enlarged ( 2 chicks added, mean brood size $4.8, \pm 0.75$ ), 9 reduced ( 2 chicks removed, $1.8 \pm 0.64$ ) and 7 control pairs (number of chicks unchanged but half of the chicks were exchanged with another brood, $2.8 \pm$ 0.64). Brood size then stayed constant in each of the treatments during the experiment (no chick mortality was detected during the growth period,-i.e., reproduction phase for the parents). Mean age in years of the parents was not significantly different among the three groups (enlarged: $2.3 \pm 0.8$, reduced: $2.1 \pm 0.8$, control: $1.7 \pm 0.9$; total range for the three groups, $1-3$ years; GLMM, $F=2.68, p=0.079$; post-hoc pair-wise comparisons: control vs. enlarged $p=0.080$, control vs. reduced $p=0.226$, 
reduced vs. enlarged $p=0.534$ ). Birds involved in the experiment had experienced at least one reproductive event prior to the brood size manipulation (no differences among groups in the number of previous breeding events, $p=0.558$ ). Four pairs were formed by partners that have been previously associated for reproduction. These pairs were distributed in the three experimental groups ( 1 in enlarged, 1 in control and 2 in the reduced group). Premanipulated clutch size did not differ between groups (enlarged: $3.8 \pm 0.4$, reduced: $4.7 \pm 0.5$, control: $3.6 \pm 0.5$; total range for the three groups, $2-7$ eggs; $p=0.078$; post-hoc pair-wise comparisons: control vs. enlarged $p=0.644$, control vs. reduced $p=$ 0.095 , reduced vs. enlarged $p=0.200)$. Pre-manipulated brood size did not differ between groups (enlarged: $2.8 \pm 0.4$, reduced: $2.5 \pm 0.4$, control: $3.7 \pm 0.4$; total range for the three groups, $1-5$ chicks; $p=0.085)$. Small blood samples $(50 \mu \mathrm{L})$ were collected from the brachial vein of the parents at the beginning of the reproductive period (when the breeding pairs were formed) and at the end of the reproductive period (when the chicks were 35 days old, which corresponds to the moment when they were separated from the parents). After reproduction, males and females were separated. Birds were put back in different sex-specific large aviaries $(1 \times 1 \times 2 \mathrm{~m}$, each containing 25 birds) placed in the same room.

In order to evaluate the long term effects of a manipulated reproductive effort on the breeding adults, we monitored the survival rate and collected a blood sample $(50 \mu \mathrm{L})$ on the birds that were still alive a year after the end of the breeding period (55\%10 individuals in the enlarged group, 43\%-6 individuals in the control group, $61 \%-11$ individuals in the reduced group). The mean age of the birds that died was 2.8 years \pm 0.5 , because of undetermined natural causes.

All blood samples were centrifuged $\left(2000 \mathrm{~g}, 10 \mathrm{~min}\right.$ at $\left.4^{\circ} \mathrm{C}\right)$, and red blood cells and plasma once separated were immediately stored at $-80^{\circ} \mathrm{C}$ until analyses.

\section{TELOMERE MEASUREMENTS}

Telomere length measurements were done before, after reproduction, and a year after the experiment. Telomere length was measured on DNA extracted from red blood cells (stored at $-20^{\circ} \mathrm{C}$ until analysis), which are nucleated in birds, using DNeasy Blood and Tissue kit (Qiagen). Telomere length was assessed by the quantitative real-time amplification (qPCR) procedure (Cawthon, 2002), previously used in zebra finches (Criscuolo et al., 2009). Relative telomere length is expressed as the ratio $(\mathrm{T} / \mathrm{S})$ of telomere repeat copy number $(\mathrm{T})$ to a control single gene copy number (S). We used glyceraldehyde3-phosphate dehydrogenase (GAPDH) as a single control gene. Forward and reverse primers for the GAPDH gene were $5^{\prime}$-AACCAGCCAAGTACGATGACAT- $3^{\prime}$ and $5^{\prime}$-CCATCA GCAGCAGCCTTCA-3' respectively. Telomere primers were: Tellb (5'-CGGTTTGTTTGGGTTTGGGTTTGGGTTTGGGTT TGGGTT- $\left.3^{\prime}\right)$ and Tel2b (5'-GGCTTGCCTTACCCTTACCCTTA CCCTTACCCTTACCCT- $\left.3^{\prime}\right)$. qPCR for both telomere and GAPDH were performed using $5 \mathrm{ng}$ of DNA with sets of primers Tel1b/Tel2b (or GAPDH-F/GAPDH-R), each used at a concentration of $200 / 200 \mathrm{nM}$, in a final volume of $10 \mu \mathrm{l}$ containing $5 \mu \mathrm{l}$ of Power SYBR Green PCR Master Mix (Applied biosystems). Telomere and GAPDH real time amplification were performed on two different plates. qPCR conditions for telomeres were $10 \mathrm{~min}$ at $95^{\circ} \mathrm{C}$ followed by 30 cycles of $1 \mathrm{~min}$ at $56^{\circ} \mathrm{C}$ and $1 \mathrm{~min}$ at $95^{\circ} \mathrm{C}$. PCR conditions for the GAPDH were $10 \mathrm{~min}$ at $95^{\circ} \mathrm{C}$ followed by 40 cycles of $1 \mathrm{~min}$ at $60^{\circ} \mathrm{C}$ and $1 \mathrm{~min}$ at $95^{\circ} \mathrm{C}$. Each plate (telomere and GAPDH) included serial dilutions $(10,5,2.5,1.25 \mathrm{ng})$ of DNA of the same reference bird. This was used to generate a reference curve to control for the amplifying efficiency of the qPCR (efficiencies for GAPDH and telomere plates were between 95 and $105 \%)$. Samples were run in duplicate on each plate. Samples were randomly distributed (for age and treatment) and run on a total of 4 plates on which samples from all groups were mixed. No plate effect was detected on telomere lengths $(p=0.455)$. To take into account the slight variation of efficiencies between telomere and GAPDH amplifications, we calculate relative telomere length using the method suggested by (Pfaffl, 2001). The relative T/S ratios were calculated using the formula: $\left[(1+\text { E telomere })^{\wedge} \Delta \mathrm{Ct}\right.$ telomere (control-sample) $/(1+\mathrm{E} \mathrm{GAPDH}) \wedge \Delta \mathrm{Ct}$ GAPDH (control-sample)], in which "control" corresponds to the value obtained for the reference sample (Pfaffl, 2001).

Mean intra-plate coefficient of variation was $1.1 \%$ for the $C t$ values of the GAPDH assays and 3.0\% for the $C t$-values of the telomere assays, and inter-plate coefficient of variation was calculated on four samples repeated on the different plates and was of $1.6 \%$ for the $C t$-values the GAPDH assays, $2.9 \%$ for the $C t$ values of the telomere assays. Mean coefficient of variation for the relative $\mathrm{T} / \mathrm{S}$ ratios was $8.5 \%$. Both a negative control (water) and melting curves were run for each plate to check for non-specific amplification and primer-dimer artefacts.

\section{OXIDATIVE STRESS MEASUREMENTS}

Oxidative stress measurements were done before, after reproduction, and a year after the experiment. We investigated oxidative stress in plasma samples (stored at $-80^{\circ} \mathrm{C}$ until analysis), with the d-ROMs and the OXY-ADSORBENT tests (Diacron International, Grosseto, Italy) as previously described in birds (Costantini et al., 2007, 2008; Beaulieu et al., 2010, 2011) and mammals (Stier et al., 2012). The OXY-ADSORBENT test measures the total antioxidant capacity of the plasma using a colorimetric determination to quantify the ability of the antioxidant barrier to cope with the oxidant action of hypochlorous acid ( $\mathrm{HOCl})$. Measurements are expressed as mmol-1 HOCl neutralized. For the OXY- ADSORBENT assay mean intra-plate coefficient of variation was of $2.2 \%$ and mean inter-plate coefficient of variation was of $7 \%$. The d-ROMs test measures the early exposure to oxidative stress by evaluating the concentration of hydroperoxide $(\mathrm{ROOH})$ which is a reactive oxygen metabolite (ROM) resulting from the attack of ROS on macromolecules (lipids, protein, DNA). The concentration of hydroperoxyde was then calculated by comparison with a standard solution whose oxidative activity on the chromogen is equivalent to the activity of $\mathrm{H} 2 \mathrm{O} 2$ ( $0.08 \mathrm{mg} \mathrm{dl}-1)$. Measurements were expressed as $\mathrm{mg}$ dl-1 $\mathrm{H} 2 \mathrm{O} 2$ equivalents. Mean intra-plate coefficient of variation was of $1.3 \%$ and mean inter-plate coefficient of variation was of $2 \%$. Procedures for both tests were performed according to manufacturer instructions.

We also investigated DNA oxidative damage through measurements of 8-hydroxy-2-deoxy Guanosine (8-OHdG), using 
the 8-OHdG EIA kit (StressMarq Biosciences Inc., Victoria, BC Canada). 8-OHdG is the by-product of oxidative damage on DNA due to reactive oxygen species (ROS) and increased levels of this marker have been associated with the ageing process (Shen and Abate-Shen, 2007). DNA was first extracted from blood cells with (DNeasy Blood and Tissue kit Qiagen) and then genomic DNA was totally digested following the instructions described in Quinlivan and Gregory 3rd (2008). DNA damage measurements are expressed in $\mathrm{pg} / \mathrm{mL}$. Mean coefficient of variation was of $12 \%$.

\section{STATISTICAL ANALYSIS}

All statistical analyses were performed using SPSS v. 18.0.

To analyse initial between-treatment differences in body mass, body size (i.e., tarsus length), body condition (expressed as the residuals of the regression between body mass and body size), telomere length, antioxidant levels, and oxidative damage levels (response variables) before the reproductive period, generalized linear mixed models (GLMM) were used with sex and brood size manipulation as fixed factors and brood identity as a random factor. Analyses show no significant effects of treatments, sex, or the interaction between treatment and sex (all $P$-values $>0.1)$.

\section{Short and long term effects of brood manipulation on body mass, telomere length, and oxidative stress}

Body mass, telomere length, antioxidant levels, and oxidative damage levels (plasmatic and DNA) were measured at the three different periods (before, at the end, and a year after the breeding period). To compare the short and long term effects of brood size manipulation on these parameters, we used GLMMs with time period (beginning, end, and a year after the reproductive period) as a repeated variable, brood identity as a random factor, and sex, brood size manipulation, as well as the interaction between the brood size manipulation and the time period as factors in the model. Parents' age and initial brood size were also added as covariates to control for their potential effects on ageing parameters. In addition to the aforementioned factors, for body mass as a response variable, the interaction between sex, and the time period was added as fixed factor. For telomere length analysis, oxidative damage levels (plasmatic and DNA), antioxidant levels, body mass, and age were added as covariates. For plasmatic and DNA oxidative damage levels analysis, the interaction between sex and the time period was added as fixed factors, as well as antioxidant levels and body mass as covariates. For antioxidant levels analysis, body mass was added as a covariate.

Other interactions between explanatory variables (brood size manipulation ${ }^{\star}$ sex; sex ${ }^{\star}$ time; brood size manipulation $*$ sex*time; depending on the response variable) were found to be nonsignificant (all $p>0.1$ ) and therefore were not included in the final models. When found to be non-significant, terms were removed sequentially from the analysis, starting with the least significant ones. The most parsimonious models are presented.

All response variables achieved a normal distribution and homoscedasticity.

To identify differences between the breeding groups and the time periods we used LSD post-hoc tests. Results are expressed as mean $\pm \mathrm{SE}$.

\section{Effects on adult survival}

We used a binomial GLMM to analyse survival a year after the breeding period where the brood size manipulation, the sex, the age and telomere length at the end of the breeding period were entered as explanatory variables. Other interactions between explanatory variables were found to be non-significant (all $p>0.1$ ) and therefore were not included in the final model.

\section{RESULTS}

\section{EFFECTS OF BROOD SIZE MANIPULATION ON NESTLINGS}

The effect of brood size manipulation had strong consequences on nestling development. Nestlings from enlarged broods stayed longer in the nest compared to those from control and reduced broods (LMM, brood size effect D.F $=2, F=18.27, p<0.001$; LSD post-hoc comparisons after a general linear model, $p=0.001$ and $p<0.001$ respectively; mean age in days at fledging: enlarged broods: $21.3 \pm 0.4$, reduced broods: $17.0 \pm 0.6$, control broods: $18.4 \pm 0.7)$. At the end of the growth period ( 35 days), nestlings from enlarged broods were lighter than those from control and reduced broods (LMM, brood size effect D.F $=2, F=10.76, p<$ 0.001; LSD post-hoc comparisons after a general linear model, $p=0.013$ and $p=0.015$ respectively; mean body mass in grams: enlarged broods: $12.1 \pm 0.3$, reduced broods: $13.6 \pm 0.5$, control broods: $13.8 \pm 0.6)$.

\section{SHORT AND LONG TERM EFFECTS OF BROOD SIZE MANIPULATION ON ADULT BODY MASS, OXIDATIVE STRESS, AND TELOMERE LENGTH}

There was no effect of brood size manipulation on adult body mass (Table 1, $p=0.466$ ). However, body mass was affected by the time period (Table 1, $p=0.026$ ), individuals being lighter during the breeding event compared to a year after the experiment (mean values of mass in grams \pm SE, before reproduction: $15.8 \pm$ 0.7 , after reproduction: $15.5 \pm 0.7$, a year after: $17.2 \pm 0.8$; LSD post-hoc comparisons, respectively $p=0.027$ and $p=0.008$ ).

All groups exhibited non-different mean plasmatic and DNA oxidative stress levels over the experiment (d-ROM: $p=0.581$; 8-oxo-dG: $p=0.756$ ) (Table 1). However, the time period (i.e., before vs. at the end vs. a year after the breeding event) as well as sex had significant effects on plasmatic oxidative stress (Table 1). Indeed, at the end of the breeding period and a year after reproduction, females exhibited higher plasmatic levels of oxidative stress than males (LSD post-hoc comparisons, respectively $p<$ 0.001 and $p=0.034$ ) (Table 1) (Figure 1). All groups exhibited similar plasmatic antioxidant levels $(p=0.700)$ (Table 1). Nonetheless, antioxidant levels were higher in all groups when measured a year after the experiment compared to the reproduction period (Table 1, time effect $p<0.001$, LSD post-hoc comparisons both $p<0.001$ ) (Figure 2).

The interaction between brood size manipulation and the time period had significant effects on telomere length (Table 1). Parents forced to raise two additional chicks had reduced telomere lengths at the end of the reproductive period compared to parents raising a control brood or a reduced brood (LSD post-hoc comparisons, $p=0.005$ and $p<0.001$, Figure 3 ). There was no significant difference of telomere length between the control and the reduced group (LSD post-hoc comparisons, $p=0.131$ ). The same pattern was observed a year after the experiment. Indeed, 
Table 1 | Results of linear mixed model on body mass, telomere length, oxidative damage levels (plasmatic measured as mg dl-1 $\mathrm{H} 2 \mathrm{O} 2$ equivalents and DNA measured as 8-OHdG pg/ml), and antioxidant levels (measured as mmol-1 $\mathrm{HOCl}$ neutralized).

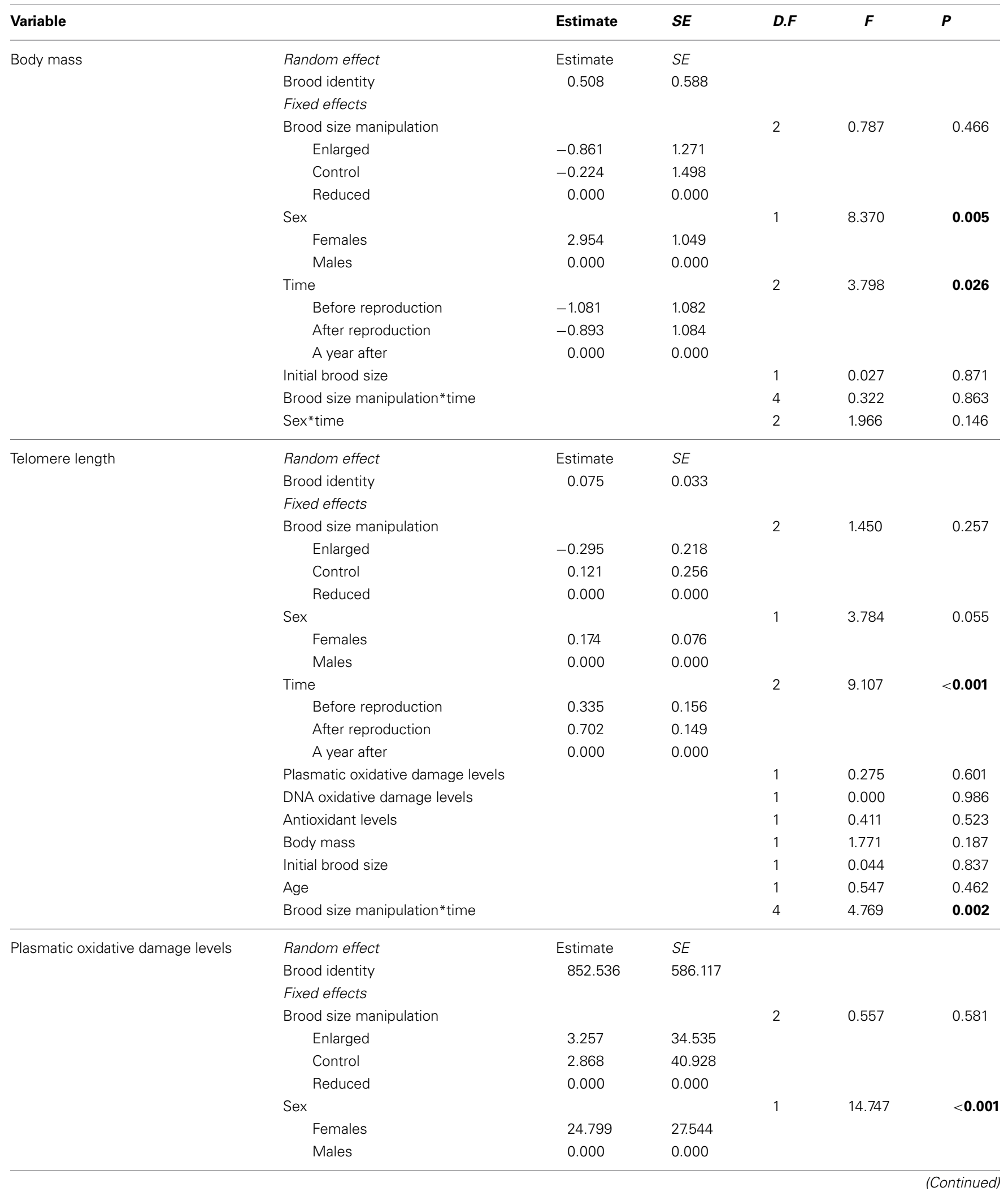


Table 1 | Continued

\begin{tabular}{|c|c|c|c|c|c|c|}
\hline \multirow[t]{8}{*}{ Variable } & & Estimate & $S E$ & D.F & $\boldsymbol{F}$ & $P$ \\
\hline & Time & & & 2 & 8.302 & $<0.001$ \\
\hline & Before reproduction & 56.918 & 29.443 & & & \\
\hline & A year after & 0.000 & 0.000 & & & \\
\hline & Antioxidant levels & & & 1 & 0.797 & 0.374 \\
\hline & Age & & & 1 & 0.324 & 0.572 \\
\hline & Brood size manipulation*time & & & 4 & 0.225 & 0.924 \\
\hline & Sex*time & & & 2 & 8.368 & $<0.001$ \\
\hline \multicolumn{7}{|c|}{ DNA oxidative damage levels } \\
\hline & Random effect & Estimate & $S E$ & & & \\
\hline & Enlarged & 23.191 & 101.257 & & & \\
\hline & Control & -113.363 & 120.608 & & & \\
\hline & Reduced & 0.000 & 0.000 & & & \\
\hline & Sex & & & 1 & 0.366 & 0.547 \\
\hline & Females & -24.198 & 85.650 & & & \\
\hline & Males & 0.000 & 0.000 & & & \\
\hline & Time & & & 2 & 1.070 & 0.347 \\
\hline & Before reproduction & -31.864 & 96.786 & & & \\
\hline & After reproduction & -17.549 & 92.139 & & & \\
\hline & A year after & 0.000 & 0.000 & & & \\
\hline & Antioxidant levels & & & 1 & 4.849 & 0.030 \\
\hline \multirow{17}{*}{ Antioxidant levels } & Brood identity & 50.245 & 152.717 & & & \\
\hline & Fixed effects & & & & & \\
\hline & Brood size manipulation & & & 2 & 0.363 & 0.700 \\
\hline & Enlarged & -21.044 & 20.008 & & & \\
\hline & Control & -12.107 & 23.885 & & & \\
\hline & Reduced & 0.000 & 0.000 & & & \\
\hline & Sex & & & 1 & 1.044 & 0.310 \\
\hline & Females & 8.094 & 7.921 & & & \\
\hline & Males & 0.000 & 0.000 & & & \\
\hline & Time & & & 2 & 8.727 & $<0.001$ \\
\hline & Before reproduction & -58.594 & 16.838 & & & \\
\hline & After reproduction & -53.902 & 16.787 & & & \\
\hline & A year after & 0.000 & 0.000 & & & \\
\hline & Body mass & & & 1 & 0.456 & 0.501 \\
\hline & Initial brood size & & & 1 & 3.434 & 0.080 \\
\hline & Age & & & 1 & 1.737 & 0.197 \\
\hline & Brood size manipulation*time & & & 4 & 1.090 & 0.366 \\
\hline
\end{tabular}

Non-significant covariates are left in the final model to show which physiological parameters affect response variables. The significant terms of the models remain so even when non-significant covariates and interactions are removed. Estimates and SE for the general linear mixed models on body mass, telomere length, oxidative damage levels, and antioxidant levels. Data from the male group, the reduced group and the year after group are used as a reference to calculate the estimates. 


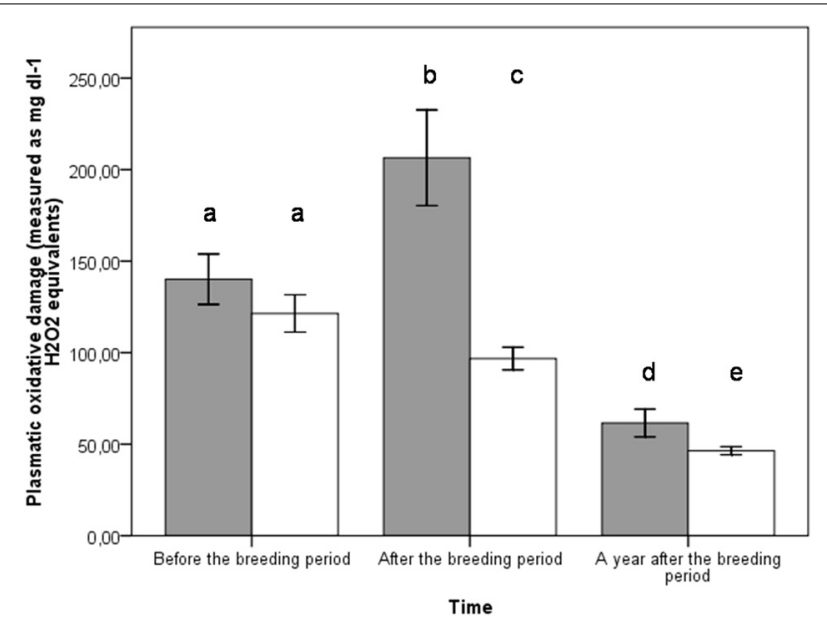

FIGURE 1 | Plasmatic oxidative damage at different time periods: before the breeding event (females $n=25$, males $n=25$ ), at the end of the breeding (females $n=25$, males $n=25$ ), a year after the breeding event (females $\boldsymbol{n}=\mathbf{1 0}$, males $\boldsymbol{n}=\mathbf{1 7}$ ). Bars represent $\pm S E$. Females in gray and males in white.

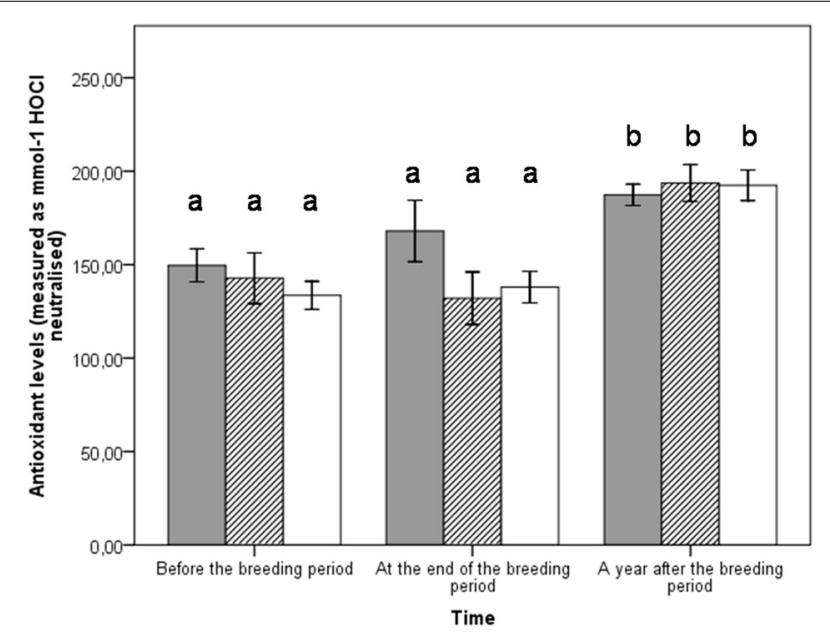

FIGURE 2 | Plasmatic antioxidant levels for both parents in the three different groups at different time periods: before the breeding event (enhanced group $n=18$, control group $n=18$, reduced group $n=14$ ), at the end of the breeding event (enhanced group $n=18$, control group $n=18$, reduced group $n=14$ ), a year after the breeding event (enhanced group $n=10$, control group $n=6$, reduced group $n=11$ ). Bars represent \pm SE. Enhanced in gray, control in hatched, reduced in white.

the detrimental effect of brood enlargement on telomere length was amplified with time and the enlarged group displayed the shortest telomeres a year after reproduction compared to the control and reduced groups (LSD post-hoc comparisons, $p=0.030$ and $p=0.022$ respectively, Figure 3). Conversely, at the same time, parents from the control and reduced groups displayed nondifferent telomere lengths (LSD post-hoc comparisons, $p=0.498$, Figure 3). There was no relationship between telomere length and plasmatic antioxidant (OXY; $\left.r^{2}=0.02, p=0.523\right)$ or oxidative

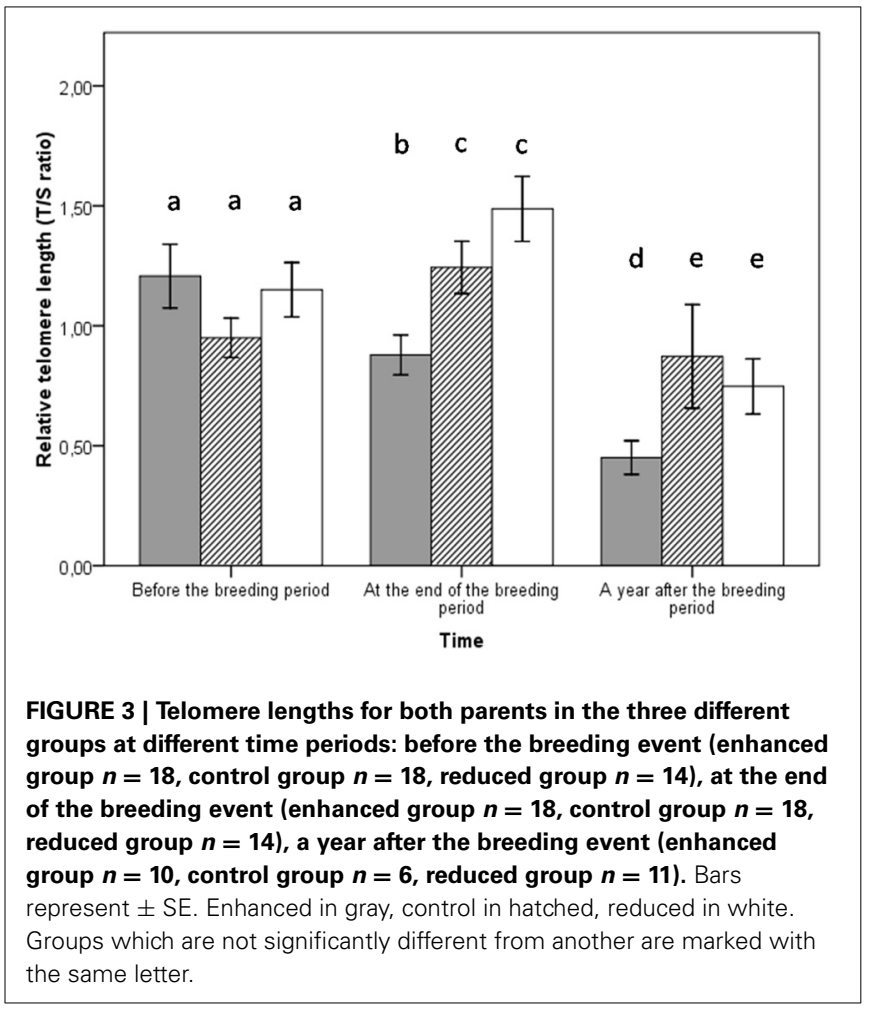

Table 2 | Results of the binomial GLMM on survival a year after the breeding event with the experimental group, sex, age, and telomere length at the end of the breeding period were entered as explanatory variables.

\begin{tabular}{llllc}
\hline Variable & & D.F & Khi2 & $\boldsymbol{P}$ \\
\hline Survival & $\begin{array}{l}\text { Effects } \\
\text { Brood size } \\
\text { manipulation }\end{array}$ & 2 & 3.007 & 0.222 \\
& Sex & 1 & 3.922 & $\mathbf{0 . 0 4 8}$ \\
& Age & 2 & 4.305 & 0.116 \\
& $\begin{array}{l}\text { Telomere length (end } \\
\text { of the breeding } \\
\text { period) }\end{array}$ & 1 & 0.289 & 0.591 \\
& & & \\
\end{tabular}

damage levels [d-ROM; $r^{2}=0.06, p=0.601$ and DNA oxidative damage (8-oxo-dG; $\left.r^{2}=0.01, p=0.986\right)$ or between telomere length and body mass $\left(r^{2}=0.007, p=0.187\right)$ (Table 1)]. We also controlled for the potential effect of age on telomere length, but there was no significant link between telomere length and age $(p=0.462)$.

\section{EFFECTS OF BROOD SIZE MANIPULATION ON SURVIVAL}

Survival rate a year after did not significantly differ among experimental groups (Table 2, $p=0.222$ ). Females had lower survival rates a year after reproduction than males (Table 2, $p=0.048$ ). Telomere length after the breeding period was not predictive of survival a year after (Table 2, $p=0.591$ ). 


\section{DISCUSSION}

(Daan et al., 1996) were the first to uncover that increased parental effort can have persistent effects on adult future fitness, even when feeding conditions were not restricted, therefore suggesting non-energetic trade-offs. In direct line with this suggestion, our study shows that a single costly reproductive event shortens adult telomeres in a way that telomeres are not restored 1 year after even though birds were maintained as non-breeders under optimal environmental and feeding conditions.

\section{DID THE EXPERIMENTAL TREATMENT INCREASE ADULT INVESTMENT IN REPRODUCTION?}

Contrary to previous studies (Daan et al., 1996; Alonso-Alvarez et al., 2004; Wiersma et al., 2004), increased reproductive effort did not induce a cost in terms of adult body mass loss in our study. However, reproduction triggered a short and mid-term rise in oxidative stress (D-ROM levels), as previously described (Alonso-Alvarez et al., 2004), but only in females and independently of the experimental treatment in our case. Despite the rise in antioxidant levels a year after the experiment, females still displayed higher plasmatic levels of oxidative damage. Still, we were unable to confirm this result while checking the effect of the experimental treatment on a DNA oxidative damage marker. Reproduction had no short or mid-term repercussions on DNA oxidative damage, underlying the importance to measure several oxidative markers before drawing definitive conclusion on oxidative costs (Costantini, 2008; Selman et al., 2012). Moreover, in our case the low sample size might prevent to obtain firm conclusions on non-significant results.

The lack of impact of our brood size manipulation on oxidative damage may be also attributed to the bleeding timing. In order to avoid impacting parents' blood volume during their reproduction and to avoid potential chick abandonment, the second blood sample, and body mass measurements were not taken during reproduction but when the chicks were independent (i.e., around 35 days). Interestingly, females still displayed higher plasmatic levels of oxidative damage than males when their chicks were 35 days old. Even though the level of plasmatic oxidative damage decreased a year after, it was still higher in females. A hypothetical explanation for this might be that, in zebra finches, even though parental care is performed by both sexes; females take a greater part of the burden than males (Zann and Bamford, 1996), which might explain the sex differences we observed. This difference in oxidative damage could be related to the lower survival rates that females exhibit a year after the reproductive event (see below).

Nestlings from the enlarged broods were lighter, and as they fledged later their period of dependency on the parents was longer than for the control and the reduced groups. These results are similar to what was found by (Dijkstra et al., 1990) showing that brood size manipulation affects current reproduction. This also suggests that in the case of enlarged broods, parents might not have been able to go beyond their physiological limits to care adequately for their increased number of chicks. Indeed, parents and offspring are expected to disagree over how long the period of parental investment should last and over the amount of parental investment that should be given (Trivers, 1974; Stearns, 1992).
Therefore, it is imaginable that parents might have invested less in their young when brood size was augmented to preserve their body maintenance, at least in terms of body mass. Nonetheless, it is interesting to note that brood size manipulation had some harmful effects on other components of adult body maintenance: telomere length.

\section{REPRODUCTIVE INVESTMENT AND TELOMERE LENGTH}

Our study suggests short- and mid-term deleterious effects of brood size manipulation on telomere length. Parents raising enlarged broods displayed shorter telomeres both at the end of the reproductive event (35 days) and 1 year after reproduction. This deleterious impact on telomere dynamics may be the result of an increased reproductive investment in enlarged broods. However, as the impact of our brood size manipulation on adult reproductive effort was not clear (lack of effects on adult body mass and oxidative damage), other explanations might be considered. Telomere loss results not only from the deleterious effect of oxidant attacks on DNA, but is balanced by repairing systems such as telomerase activity (Greider and Blackburn, 1985). Therefore, a possibility is that telomere erosion is not directly related to increased parental energy expenditure (Daan et al., 1996) and to an associated rise in oxidative damage. One hypothesis is that an indirect link, e.g., through a perceived stressful situation (through increased social stress within the pair and/or with the offspring) may take place (see Haussmann and Marchetto, 2010; Blackburn and Epel, 2012) for stress vs. telomere erosion relationship. Ultimately, such a social stress may have affected telomere erosion rate through a decreased telomerase activity, a topic that has been previously pointed out in humans as a result of stressful environments (Epel et al., 2004).

Surprisingly, we were unable to clearly establish a link between high levels of plasmatic oxidative damage or of DNA oxidative damage and a higher rate of telomere shortening. Firstly, even though females of all groups displayed higher plasmatic oxidative damage, their telomere loss was not exacerbated and we did not find any correlation between telomere loss and oxidative damage at the end of the reproductive event or a year after the reproductive event. These findings support the idea that the link between oxidative stress and telomere erosion is unsettled at present in vivo (Chen et al., 2001). However, beside the fact that we may have failed to detect an oxidative unbalance in adults caring for enlarged broods (see above), the lack of apparent relationship between oxidative damage and telomere loss may also be explained by other factors. In fact, our higher telomere loss in "enlarged breeders" may rather reflect the inability of birds, which were forced to raise more chicks, to insure adequate telomere maintenance; mediated either by telomerase activity (Greider and Blackburn, 1985), or the pattern of expression of protecting shelterin proteins (De Lange, 2005). However, more experimental work is needed to understand accurately how telomere maintenance mechanisms might be involved into reproductive costs.

Another noteworthy point is that the effects of brood size manipulation found on telomeres at the end of the reproductive period persist a year after the experiment. Indeed, parents subjected to increased breeding constraints had the shortest telomeres even a year after the costly reproductive effort. These results 
enrich the recent finding of long-term telomere changes over the entire reproductive life of zebra finches, showing that an erosion of telomeres takes place independently of the number of reproductive events (Heidinger et al., 2012). This latter study actually suggests that entering the reproductive life is associated to a reduction of telomere length, as a consequence of the triggering of processes leading to sexual maturity. It will be interesting to test in future experiments the impact of the progressive rise in sexual steroids on telomere dynamics. However, in (Heidinger et al., 2012) study, the breeding pairs were only allowed to produce and incubate the eggs, and not to raise any chick. Therefore, we think that both components of the reproductive costs (reproductive maturity and investment) are modulating adult telomere loss, and that chick brooding and feeding activities may have an important impact on adult cell senescence pathways (Bauch et al., 2013).

After the breeding attempt, parents of the control and the reduced groups did not incur any cost in terms of telomere loss. In fact, telomere length increased slightly. This result indicates that in optimal conditions individuals probably may adjust their reproductive effort in order to avoid any potential costs that might be associated with increased reproductive effort, such as accelerated ageing rate, potentially through activation of telomere maintenance mechanisms such as telomerase. (Haussmann et al., 2007) study might give support to this hypothesis by showing that telomerase activity is maintained throughout lifespan in different somatic tissues (including bone marrow) in birds. Therefore, telomerase activity in the hematopoietic cells of the bone marrow (Broccoli et al., 1995) might have maintained telomere length in the control and the reduced groups. In addition, when parents see their initial investment reduced (as it is the case in the reduced group), there is no apparent positive impact on telomere loss, reinforcing the idea of a fine tuned parental effort related to individual physiological state.

Our data suggest that brood size manipulation can have short and long terms effects on telomere dynamics. This is likely to have different implications at the fitness level, particularly if those results apply in natural conditions [see Daan et al. (1996) for correlative support in the wild]. Several studies indicate that, telomere length being a good predictor of survival and lifespan in wild species (Haussmann et al., 2005; Bize et al., 2009; Salomons et al., 2009; Heidinger et al., 2012), short telomeres after a costly breeding event might compromise future survival. In this context, telomere dynamics could still be a good candidate to explain the mechanistic link between reproduction and survival, but we still lack a definitive demonstration of the causality of such an ultimate consequence. Indeed, although we observe a trend for a higher mortality rate in the enlarged group (44\% mortality), we found that the level of breeding effort did not influence short term survival even for the parents that had the shortest telomeres. This trend might have been found significant had we used a larger sample size. However, these findings are consistent with those of (Heidinger et al., 2012) who also suggest that the level of breeding effort does not influence survival. A possible explanation for that would be that the study was conducted in captivity and in ad libitum feeding conditions, thus creating favorable conditions which might have preserved individuals.
Moreover, following survival rate of adult zebra finches over a year is probably not long enough to conclude. However, an interesting observation was that females exhibited higher oxidative damage and lower survival rates 1 year later, suggesting more rapid fitness consequences of an acute unexpected oxidative disorder. Such an observation between oxidative damage and survival rate was observed in European shags (Phalacrocorax aristotelis) (Noguera et al., 2012). Antioxidant levels or cell resistance to oxidative stress have also been linked to annual survival rate in free-living barn swallows (Hirundo rustica) (Saino et al., 2011) and male alpine swifts (Apus melba; Bize et al., 2008) respectively, suggesting as well an important role of oxidative balance in immediate individual fitness modulation.

In conclusion, our study highlights that brood size manipulation is related to adult long term eroded telomeres, but with no clear-cut short-term impact on adult reproductive effort (i.e., adult body mass loss or oxidative damage) and adult survival rate over a year. In the future, particular attention should be given to experimental brood manipulation and their repercussions on social interaction and stress in order to understand how telomere maintenance mechanisms may be involved into parental reproductive investment and their potential costs.

\section{AUTHOR CONTRIBUTIONS}

Conception and design of the experiment was done by Sophie Reichert, Sylvie Massemin, François Criscuolo, and Pierre Bize. Data was acquired by Sophie Reichert, Sylvie Massemin, François Criscuolo, Antoine Stier, and Sandrine Zahn while analysis and interpretation of the data was done by Sophie Reichert, François Criscuolo, Sylvie Massemin, Pierre Bize, and Antoine Stier.

\section{ACKNOWLEDGMENTS}

We thank Dr Etienne Challet, Dr Stéphane Blanc, Dr AM Thierry and Dr Neil Metcalfe for helpful discussions on the manuscript. This work was supported by the CNRS and the Conseil Régional of Alsace. The study complied with the "Principles of Animal Care" publication no. 86-23, revised 1985 of the National Institute of Health, and with current legislation (L87-848) on animal experimentation in France. This work was entirely supported by a CNRS funding and a PhD grant from the Region Alsace. We thank Hranitsky Aurélie for her help with the bird husbandry.

\section{REFERENCES}

Allsopp, R. C., Vaziri, H., Patterson, C., Goldstein, S., Younglai, E. V., Futcher, A. B., et al. (1992). Telomere length predicts replicative capacity of human fibroblasts. Proc. Natl. Acad. Sci. U.S.A. 89, 10114-10118. doi: 10.1073/pnas.89.21.10114

Alonso-Alvarez, C., Bertrand, S., Devevey, G., Prost, J., Faivre, B., and Sorci, G. (2004). Increased susceptibility to oxidative stress as a proximate cost of reproduction. Ecol. Lett. 7, 363-368. doi: 10.1111/j.1461-0248.2004.00594.x

Bauch, C., Becker, P. H., and Verhulst, S. (2013). Telomere length reflects phenotypic quality and costs of reproduction in a long-lived seabird. Proc. Biol. Sci. 280:20122540. doi: 10.1098/rspb.2012.2540

Beaulieu, M., Reichert, S., Le Maho, Y., Ancel, A., and Criscuolo, F. (2011) Oxidative status and telomere length in a long-lived bird facing a costly reproductive event. Funct. Ecol. 25, 577-585. doi: 10.1111/j.1365-2435.2010.01825.x

Beaulieu, M., Ropert-Coudert, Y., Le Maho, Y., Ancel, A., and Criscuolo, F. (2010) Foraging in an oxidative environment: relationship between $\delta 13 \mathrm{C}$ values and oxidative status in Adélie penguins. Proc. R. Soc. B Biol. Sci. 277, 1087-1092. doi: $10.1098 /$ rspb.2009.1881 
Bize, P., Criscuolo, F., Metcalfe, N. B., Nasir, L., and Monaghan, P. (2009). Telomere dynamics rather than age predict life expectancy in the wild. Proc. R. Soc. B Biol. Sci. 276, 1679-1683. doi: 10.1098/rspb.2008.1817

Bize, P., Devevey, G., Monaghan, P., Doligez, B., and Christie, P. (2008). Fecundity and survival in relation to resistance to oxidative stress in a free-living bird. Ecology 89, 2584-2593. doi: 10.1890/07-1135.1

Blackburn, E. H. (1991). Structure and function of telomeres. Nature 350, 569-573. doi: $10.1038 / 350569 \mathrm{a} 0$

Blackburn, E. H. (2000). Telomere states and cell fates. Nature 408, 53-56. doi: $10.1038 / 35040500$

Blackburn, E. H., and Epel, E. S. (2012). Telomeres and adversity: too toxic to ignore. Nature 490, 169-171. doi: 10.1038/490169a

Broccoli, D., Young, J. W., and de Lange, T. (1995). Telomerase activity in normal and malignant hematopoietic cells. Proc. Natl. Acad. Sci. U.S.A. 92, 9082-9086. doi: 10.1073/pnas.92.20.9082

Brown-Borg, H. M. (2007). Hormonal regulation of longevity in mammals. Ageing Res. Rev. 6, 28-45. doi: 10.1016/j.arr.2007.02.005

Cawthon, R. M. (2002). Telomere measurement by quantitative PCR. Nucleic Acids Res. 30:e47. doi: 10.1093/nar/30.10.e47

Cawthon, R. M., Smith, K. R., O’Brien, E., Sivatchenko, A., and Kerber, R. A. (2003). Association between telomere length in blood and mortality in people aged 60 years or older. Lancet 361, 393-395. doi: 10.1016/S0140-6736(03) 12384-7

Chen, Q. M., Prowse, K. R., Tu, V. C., Purdom, S., and Linskens, M. H. K. (2001). Uncoupling the senescent phenotype from telomere shortening in hydrogen peroxide-treated fibroblasts. Exp. Cell Res. 265, 294-303. doi: 10.1006/excr.2001.5182

Costantini, D. (2008). Oxidative stress in ecology and evolution: lessons from avian studies. Ecol. Lett. 11, 1238-1251. doi: 10.1111/j.1461-0248.2008.01246.x

Costantini, D., Cardinale, M., and Carere, C. (2007). Oxidative damage and anti-oxidant capacity in two migratory bird species at a stop-over site. Comp. Biochem. Physiol. C Toxicol. Pharmacol. 144, 363-371. doi: 10.1016/j.cbpc.2006.11.005

Costantini, D., Dell'Ariccia, G., and Lipp, H. P. (2008). Long flights and age affect oxidative status of homing pigeons (Columba livia). J. Exp. Biol. 211, 377-381. doi: 10.1242/jeb.012856

Criscuolo, F., Bize, P., Nasir, L., Metcalfe, N. B., Foote, C. G., Griffiths, K., et al. (2009). Real-time quantitative PCR assay for measurement of avian telomeres. J. Avian Biol. 40, 342-347. doi: 10.1111/j.1600-048X.2008.04623.x

Daan, S., Deerenberg, C., and Dijkstra, C. (1996). Increased daily work precipitates natural death in the kestrel. J. Anim. Ecol. 65, 539-544. doi: 10.23 $07 / 5734$

De Lange, T. (2005). Shelterin: the protein complex that shapes and safeguards human telomeres. Genes Dev. 19, 2100-2110. doi: 10.1101/gad.1346005

De Lange, T. (2009). How telomeres solve the end-protection problem. Science 326, 948-952. doi: 10.1126/science.1170633

Dijkstra, C., Bult, A., Bijlsma, S., Daan, S., Meijer, T., and Zijlstra, M. (1990). Brood size manipulations in the kestrel (Falco tinnunculus): effects on offspring and parent survival. J. Anim. Ecol. 59, 269-285. doi: 10.2307/5172

Epel, E. S., Blackburn, E. H., Lin, J., Dhabhar, F. S., Adler, N. E., Morrow, J. D., et al. (2004). Accelerated telomere shortening in response to life stress. Proc. Natl. Acad. Sci. U.S.A. 101, 17312-17315. doi: 10.1073/pnas.0407162101

Finkel, T., and Holbrook, N. J. (2000). Oxidants, oxidative stress and the biology of ageing. Nature 408, 239-247. doi: 10.1038/35041687

Greider, C. W., and Blackburn, E. H. (1985). Identification of a specific telomere terminal transferase activity in tetrahymena extracts. Cell 43, 405-413. doi: 10.1016/0092-8674(85)90170-9

Hall, M. E., Nasir, L., Daunt, F., Gault, E. A., Croxall, J. P., Wanless, S., et al. (2004). Telomere loss in relation to age and early environment in long-lived birds. Proc. R. Soc. B Biol. Sci. 271, 1571-1576. doi: 10.1098/rspb.2004.2768

Harley, C. B., Futcher, A. B., and Greider, C. W. (1990). Telomeres shorten during ageing of human fibroblasts. Nature 345, 458-460. doi: 10.1038/34 $5458 \mathrm{a} 0$

Harshman, L. G., and Zera, A. J. (2007). The cost of reproduction: the devil in the details. Trends Ecol. Evol. 22, 80-86. doi: 10.1016/j.tree.2006.10.008

Haussmann, M. F., Longenecker, A. S., Marchetto, N. M., Juliano, S. A., and Bowden, R. M. (2011). Embryonic exposure to corticosterone modifies the juvenile stress response, oxidative stress and telomere length. Proc. R. Soc. B Biol. Sci. 279, 1447-1456. doi: 10.1098/rspb.2011.1913
Haussmann, M. F., and Marchetto, N. M. (2010). Telomeres: linking stress and survival, ecology and evolution. Curr. Zool. 56, 714-727.

Haussmann, M. F., Winkler, D. W., Huntington, C. E., Nisbet, I. C. T., and Vleck, C. M. (2007). Telomerase activity is maintained throughout the lifespan of long-lived birds. Exp. Gerontol. 42, 610-618. doi: 10.1016/j.exger.2007. 03.004

Haussmann, M. F., Winkler, D. W., O’Reilly, K. M., Huntington, C. E., Nisbet, I. C. T., and Vleck, C. M. (2003). Telomeres shorten more slowly in long-lived birds and mammals than in short-lived ones. Proc. R. Soc. B Biol. Sci. 270, 1387-1392. doi: $10.1098 / \mathrm{rspb} .2003 .2385$

Haussmann, M. F., Winkler, D. W., and Vleck, C. M. (2005). Longer telomeres associated with higher survival in birds. Biol. Lett. 1, 212-214. doi: 10.1098/rsbl.2005.0301

Heidinger, B. J., Blount, J. D., Boner, W., Griffiths, K., Metcalfe, N. B., and Monaghan, P. (2012). Telomere length in early life predicts lifespan. Proc. Natl. Acad. Sci. U.S.A. 109, 1743-1748. doi: 10.1073/pnas.1113306109

Kotrschal, A., Ilmonen, P., and Penn, D. J. (2007). Stress impacts telomere dynamics. Biol. Lett. 3, 128-130. doi: 10.1098/rsbl.2006.0594

Metcalfe, N. B., and Monaghan, P. (2013). Does reproduction cause oxidative stress? An open question. Trends Ecol. Evol. 28, 347-350. doi: 10.1016/j.tree.2013.01.015

Min, K. J., Lee, C. K., and Park, H. N. (2012). The lifespan of Korean eunuchs. Curr. Biol. 22, R792-R793. doi: 10.1016/j.cub.2012.06.036

Monaghan, P., and Haussmann, M. F. (2006). Do telomere dynamics link lifestyle and lifespan? Trends Ecol. Evol. 21, 47-53. doi: 10.1016/j.tree.2005.11.007

Monaghan, P., Metcalfe, N. B., and Torres, R. (2009). Oxidative stress as a mediator of life history trade-offs: mechanisms, measurements and interpretation. Ecol. Lett. 12, 75-92. doi: 10.1111/j.1461-0248.2008.01258.x

Noguera, J. C., Kim, S.-Y., and Velando, A. (2012). Pre-fledgling oxidative damage predicts recruitment in a long-lived bird. Biol. Lett. 8, 61-63. doi: 10.1098/rsbl.2011.0756

Partridge, L., and Farquhar, M. (1981). Sexual activity reduces lifespan of male fruitflies. Nature 294, 580-582. doi: 10.1038/294580a0

Pauliny, A., Wagner, R. H., Augustin, J., Szép, T., and Blomqvist, D. (2006). Ageindependent telomere length predicts fitness in two bird species. Mol. Ecol. 15, 1681-1687. doi: 10.1111/j.1365-294X.2006.02862.x

Pfaffl, M. W. (2001). A new mathematical model for relative quantification in realtime RT-PCR. Nucleic Acids Res. 29:e45. doi: 10.1093/nar/29.9.e45

Quinlivan, E. P., and Gregory, J. F. 3rd. (2008). DNA digestion to deoxyribonucleoside: a simplified one-step procedure. Anal. Biochem. 373, 383-385. doi: 10.1016/j.ab.2007.09.031

Saino, N., Caprioli, M., Romano, M., Boncoraglio, G., Rubolini, D., Ambrosini, R., et al. (2011). Antioxidant defenses predict long-term survival in a passerine bird. PLoS ONE 6:e19593. doi: 10.1371/journal.pone.0019593

Salomons, H. M., Mulder, G. A., van de Zande, L., Haussmann, M. F., Linskens, M. H. K., and Verhulst, S. (2009). Telomere shortening and survival in free-living corvids. Proc. R. Soc. B Biol. Sci. 276, 3157-3165. doi: 10.1098/rspb.2009.0517

Santos, E. S. A., and Nakagawa, S. (2012). The costs of parental care: a meta-analysis of the trade-off between parental effort and survival in birds. J. Evol. Biol. 25, 1911-1917. doi: 10.1111/j.1420-9101.2012.02569.x

Selman, C., Blount, J. D., Nussey, D. H., and Speakman, J. R. (2012). Oxidative damage, ageing, and life-history evolution: where now? Trends Ecol. Evol. 27, 570-577. doi: 10.1016/j.tree.2012.06.006

Shen, M. M., and Abate-Shen, C. (2007). Pten Inactivation and the emergence of androgen-independent prostate cancer. Cancer Res. 67, 6535-6538. doi: 10.1158/0008-5472.CAN-07-1271

Smith, J. M. (1958). The effects of temperature and of egg-laying on the longevity of drosophila subobscura. J. Exp. Biol. 35, 832-842.

Speakman, J. R. (2008). The physiological costs of reproduction in small mammals. Philos. Trans. R. Soc. B Biol. Sci. 363, 375-398. doi: 10.1098/rstb.2007.2145

Stearns, S. (1992). The Evolution of Life Histories (POD). Oxford: Oxford University Press.

Stier, A., Reichert, S., Massemin, S., Bize, P., and Criscuolo, F. (2012). Constraint and cost of oxidative stress on reproduction: correlative evidence in laboratory mice and review of the literature. Front. Zool. 9:37. doi: 10.1186/17429994-9-37

Tavecchia, G., Coulson, T., Morgan, B. J. T., Pemberton, J. M., Pilkington, J. C., Gulland, F. M. D., et al. (2005). Predictors of reproductive cost in female Soay sheep. J. Anim. Ecol. 74, 201-213. doi: 10.1111/j.1365-2656.2005.00916.x 
Trivers, R. L. (1974). Parent-offspring conflict. Am. Zool. 14, 249-264. doi: $10.1093 / \mathrm{icb} / 14.1 .249$

Von Zglinicki, T. (2002). Oxidative stress shortens telomeres. Trends Biochem. Sci. 27, 339-344. doi: 10.1016/S0968-0004(02)02110-2

Wiersma, P., Selman, C., Speakman, J. R., and Verhulst, S. (2004). Birds sacrifice oxidative protection for reproduction. Proc. R. Soc. B Biol. Sci. 271, S360-S363. doi: 10.1098/rsbl.2004.0171

Zann, R. A., and Bamford, M. (1996). The Zebra Finch: A Synthesis of Field and Laboratory Studies. Oxford: Oxford University Press.

Conflict of Interest Statement: The authors declare that the research was conducted in the absence of any commercial or financial relationships that could be construed as a potential conflict of interest.
Received: 13 January 2014; accepted: 22 March 2014; published online: 22 April 2014. Citation: Reichert S, Stier A, Zahn S, Arrivé M, Bize P, Massemin S and Criscuolo F (2014) Increased brood size leads to persistent eroded telomeres. Front. Ecol. Evol. 2:9. doi: 10.3389/fevo.2014.00009

This article was submitted to Behavioral and Evolutionary Ecology, a section of the journal Frontiers in Ecology and Evolution.

Copyright (c) 2014 Reichert, Stier, Zahn, Arrivé, Bize, Massemin and Criscuolo. This is an open-access article distributed under the terms of the Creative Commons Attribution License (CC BY). The use, distribution or reproduction in other forums is permitted, provided the original author(s) or licensor are credited and that the original publication in this journal is cited, in accordance with accepted academic practice. No use, distribution or reproduction is permitted which does not comply with these terms. 